\title{
FASE EXPLORATORIA DE LA FORMULACIÓN DE UN MODELO DE RESPONSABILIDAD SOCIAL PARA LA UNIVERSIDAD MILITAR NUEVA GRANADA
}

\section{Exploratory phase of formulation of a social responsibility model for the Universidad Militar Nueva Granada}

\author{
Anny Astrid Espitia Cubillos ${ }^{1}$, Sergio Raúl Quintero Rodríguez ${ }^{2}$ \\ 1-2Facultad de Ingeniería, Universidad Militar Nueva Granada, (Bogotá D.C, Colombia). \\ Email: 1anny.espitia@unimilitar.edu.co, ${ }^{2}$ sergquintero@gmail.com
}

(Recibido Junio 22 de 2019 y aceptado Marzo 11 de 2020)

\section{Resumen}

La explotación de los recursos naturales ha sido un proceso imparable a partir de la industrialización, incrementado a su paso factores como los problemas ambientales y sociales a nivel mundial; a partir de lo anterior, surge a nivel global, y en especial en el sector productivo, la preocupación por mejorar dicha situación, y la urgencia por diseñar y poner en marcha acciones que promuevan el desarrollo sostenible, mediante las cuales se favorezca la integración del contexto con cada una de las organizaciones, y así generar impactos positivos en la sociedad tanto a nivel local como a nivel mundial, situación que también le concierne a las instituciones educativas. Para responder a esta realidad, la Universidad Militar Nueva Granada desea formular su modelo de Responsabilidad Social Universitaria partiendo de la revisión de las acciones de otras Instituciones de Educación Superior públicas, acción que permite identificar las estrategias planteadas y dar respuesta a su propósito de formar profesionales con responsabilidad social y desarrollar sus funciones misionales bajo esta misma premisa. Para ello se determinó el avance a nivel económico, legal, ético y discrecional documentado y publicado por algunas de las Universidades que integran el Sistema Universitario Estatal (SUE) y se comparó con el estado actual de la Universidad objeto de estudio para contar con algunos referentes que sirvan como punto de partida para la formulación de un modelo de responsabilidad social propio.

Palabras clave: ética, responsabilidad social universitaria, universidad pública, fase exploratoria, educación.

\begin{abstract}
Natural resources exploitation has been an unstoppable process since industrialization, increasing in its wake factors such as environmental and social problems worldwide; taking this as a starting point, there is a global level, and especially in the productive sector, concern to improve this situation, and urgency to design and implement actions that promote sustainable development, through which integration is fostered the context with each of the organizations, and thus generate positive impacts on society both locally and globally. This situation also concerns educational institutions. To give an answer to this reality, the Universidad Militar Nueva Granada wants to formulate their model of social university responsibility, starting off with the revision of the actions of other higher public education institutions, that allows to identify the generated strategies and give answer to their propose of coaching professionals whit social responsibility and develop their missional functions under that same premise. For this purpose, progress was made at an economic, legal, ethical and discretional level documented and published by some of the Universities that make up the State University System (SUE) and compared with the current state of the University under study to have some references to serve as a starting point for the formulation of own University Social Responsibility.
\end{abstract}


Key words: ethics, university social responsibility, public university, exploratory phase, education.

\section{INTRODUCCIÓN}

El objetivo de este documento es mostrar la fase inicial del proyecto de investigación "Diseño de un modelo de Responsabilidad Social universitaria para la Universidad Militar Nueva Granada". En esta fase denominada exploratoria, se llevó a cabo los procesos de levantamiento y organización de información para poder ejecutar un proceso comparativo en el actuar de las diferentes instituciones de educación superior (IES) públicas con referencia a la Responsabilidad Social Universitaria (RSU), y poder tomar los elementos de mayor relevancia a considerar en el planteamiento del modelo para la Universidad Militar Nueva Granada (UMNG).

Las organizaciones entendidas como componentes sociales en la comunidad, tienen la obligación moral de retribuir el beneficio que adquieren de la comunidad con la cual interactúan. Esto quiere decir que los esfuerzos dentro de las organizaciones no se deben enfocar solamente en la obtención de utilidades económicas, sino que las acciones y el actuar debe incluir dentro de sus objetivos estratégicos, elementos que generen beneficio a todos los sectores de la comunidad en aspectos económicos, ambientales y todos aquellos que signifiquen mejoramiento de las condiciones del contexto.

Las entidades de educación superior, se entienden como organizaciones cuyo objetivo principal es la formación de individuos capaces de desenvolverse de manera competitiva y dar soluciones innovadoras a las diferentes problemáticas de la sociedad. J.R García [1] trasmite la necesidad de formar ciudadanos con carácter crítico y coherentes en su actuar con las condiciones culturales que corresponden a la realidad y contexto histórico de cada individuo.

Para cumplir con el objetivo de formación superior y la obligación moral ya señalada, la UMNG ya ha considerado dentro de su planeación estratégica la responsabilidad social, por un lado, en su misión señala “(...) el fin de formar ciudadanos íntegros y socialmente responsables que promuevan la justicia, la equidad, el respeto por los valores humanos y contribuyan al progreso del sector Defensa y a la sociedad en general." [2], así mismo su Visión declara que "será reconocida (...); en cumplimiento de la responsabilidad social, que le permita anticipar, proponer y desarrollar soluciones que respondan a las necesidades de la sociedad y del sector Defensa" [2], finalmente, en el plan de desarrollo incluye el numeral 6.4 responsabilidad social [3], en donde se plantea la implementación de la responsabilidad social universitaria, a nivel institucional; de tal forma, que se garantice su transversalidad, con la participación de todos los integrantes de la comunidad educativa y el contexto mismo de la universidad.

Sin desconocer la existencia de propuestas de modelos genéricos de RSU aplicables a las IES [4], la iniciativa de crear un modelo propio adaptado a las particularidades de la UMNG, en concordancia con [3], busca dar respuesta a la sociedad creando bases para la formación de profesionales con la capacidad responder profesionalmente en sus áreas de formación, sin dejar de lado la importancia de la labor social que desempeñan dentro de la comunidad y materializar la planeación estratégica institucional. Los resultados presentados en el presente documento corresponden exclusivamente a la fase exploratoria que tiene como finalidad contrastar la actuación de las instituciones de educación superior (IES) públicas con referencia a la Responsabilidad Social Universitaria (RSU) con el estado actual de la Universidad Militar Nueva Granada, para tener un punto de partida para el futuro planteamiento del modelo propio para la Universidad Militar Nueva Granada (UMNG) que responda a las particularidades de la misma y que sirva de orientación a otras instituciones del SUE para el desarrollo del mismo proceso.

\section{METODOLOGÍA}

Para la fase exploratoria, se realizó un análisis comparativo, teniendo inicialmente como criterios, las categorías 
propuestas específicamente para el tema de responsabilidad social universitaria por [4], a saber:

1. Campus responsable, en la cual se plantea la necesidad de contar con un clima laboral y educativo que propicie y promueva un actuar responsable con la sociedad y el medio ambiente por parte de todos los integrantes de la institución.

2. Formación profesional y ciudadana, que señala el diseño curricular debe enfocase en la formación y fomento de competencias de servicio social.

3. Participación social, que propone se direccionen los procesos universitarios hacia la integración y participación de proyectos conjuntos con la comunidad, de tal forma, que se propicie su desarrollo.

4. Gestión social del conocimiento, que busca tramitar socialmente el componente investigativo de la universidad. En este sentido, los procesos de este factor deben estar alineados con el desarrollo local y nacional de acuerdo a los programas sociales que se plantean en los gobiernos.

En la segunda etapa de la fase exploratoria, se llevó a cabo el mismo tipo de análisis comparativo teniendo como base las cuatro categorías de responsabilidad social propuestas por J.P Sulbarán [5] en diferentes organizaciones, y lo propuesto por Bokhari [6], quien afirma existe un consenso en las definiciones de responsabilidad social, puesto que esta no debe estar ligada solamente al aspecto económico, sino que debe extenderse a aspectos ambientales, y problemáticas ligadas a la comunidad.

El autor [6] coincide con las categorías propuestas por Sulbarán [5], vigentes en la actualidad, a saber:

1. En primera instancia se encuentra la categoría económica, en la cual se entienden las responsabilidades de la organización con sus clientes tanto internos como externos en su retribución económica y generación de riqueza para su contexto.

2. Toda acción que lleva a cabo la organización tiene una repercusión legal, por lo tanto, para esto se cuenta con la segunda categoría, en la cual las acciones de la organización deben estar ubicadas dentro de las leyes que se dictan en el contexto organizacional.

3. La tercera categoría se refiere a la ética con que la organización planea y ejecuta sus actividades en la sociedad, todas las decisiones que se tomen tienen repercusiones, y a pesar de que algunas de estas no estén codificadas dentro de la sociedad con leyes o normas, si tienen aceptación positiva o negativa dentro de la misma, lo que implica que la organización debe conocer cuales tiene aceptación para poder tomar las mejores decisiones.

4. Como última categoría se encuentra la discrecional, en la cual se identifican las intenciones de los directivos de la compañía en realizar cambios para el mejoramiento de las condiciones del entorno, sin necesidad de cumplir con las leyes o reglamentación que le exige la sociedad.

En el particular para la RSU, Bokhari [6], quien toma como referente a Vallaeys, afirma que el proceso de formación de profesionales debe estar enfocado en todos los niveles institucionales, por lo que, en las universidades, es necesario que las categorías anteriores sean transversales.

Para dichas categorías, se analizó lo documentado y divulgado en artículos publicados en bases de datos científicas por parte de algunas de las Universidades que integran el Sistema Universitario Estatal (SUE), el cual está integrado por las 32 universidades estatales, y en el que se formalizan compromisos para la oferta académica orientada a fortalecer la etapa de posconflicto en cada una de las regiones a las cuales pertenece cada institución. Las funciones del SUE se establecen en la Ley 30 de 1992, artículo 81 , y se definen tres objetivos principales enfocados en la optimización de los recursos públicos mejorando la calidad educativa, teniendo como eje principal el intercambio de experiencias y conocimientos de estudiantes y docentes, y el apoyo en el diseño de estrategias que propendan por el mejoramiento continuo del sistema, haciendo uso de herramientas evaluativas tanto para las instituciones como para el sistema en general [7]. 
Es importante considerar que, en el año 2016 los rectores de Universidades Públicas que integran el SUE, determinaron el compromiso de orientar las actividades y esfuerzos pedagógicos hacia el fortalecimiento de los procesos de paz que se adelantaban, en ese momento y, que se adelanten por parte del gobierno con grupos al margen de la ley, tal como fue declarado en el comunicado de compromiso con el proceso de paz [8], teniendo en cuenta la autonomía institucional y la labor social que ejecutan las universidades como formadoras de profesionales.

Sumado a esto, se formalizó el compromiso de hacer acompañamiento pedagógico a los procesos de paz, así como orientar las cátedras de cada una de las regiones hacia la paz, el posconflicto y la formación de ciudadanos con valores que permitan mantener una paz duradera en el país. Pese a ello, los hallazgos permiten afirmar que no todas las instituciones del SUE han documentado y publicado sus avances al respecto, se comparan los avances presentados por las Universidades de: Antioquia, Atlántico, Caldas, Cauca, La Gu ajira, Nacional y Nariño, con lo ejecutado por la UMNG.

En general, teniendo en cuenta que los conceptos de Responsabilidad Social Empresarial y Responsabilidad Social Universitaria no son excluyentes, y por el contrario pueden ser complementarios, se propone hacer un análisis comparativo matricial como punto de partida para la construcción de modelos propios que integre las categorías, usando la estructura que se presenta en la Tabla 1.

\section{RESULTADOS}

A continuación, se muestra el resultado del estudio bibliográfico para cada una de las categorías, y los hallazgos de universidades que integran el SUE, para guiar el proceso de diseño del modelo de responsabilidad social para la UMNG.

\subsection{Económica}

Para este aspecto, las universidades coinciden en la necesidad de mantener contacto permanente con el estado, la sociedad y el sector productivo, actuando como punto de enlace entre estos actores y formando profesionales que promuevan impactos económicos significativos que contribuyan al mejoramiento de las condiciones de los sectores de la sociedad.

El reto que se reconoce por parte de las instituciones, se encuentra en alinear las metas y proyecciones de los estudiantes frente a lo que requiere la comunidad con la cual interactúan, por lo tanto, la formación de estos debe enfocarse en que las acciones adelantadas en el sector productivo signifiquen escalamiento social y mejoramiento de condiciones de vida para todos los integrantes de la comunidad.

En el contexto nacional de descentralización de la educación superior, las instituciones reconocen la importancia de contar con un programa el cual sea de alcance máximo para la región en la cual ejercen su labor. La cobertura de la oferta educativa juega un papel importante, puesto que, para continuar transformando la realidad económica de las regiones, se debe tener cobertura en la mayor extensión de territorio posible, aunque el presupuesto no sea suficiente. Es por esta razón, que en cada región hay universidades que ofrecen programas de acuerdo a las necesidades de la zona de impacto en la cual se encuentran.

Todos los esfuerzos que se llevan a cabo en las universidades se encuentran incluidos en los planes de desarrollo institucionales, garantizando que el cumplimiento de estas políticas sea efectivo durante el periodo considerado en la planeación institucional.

De las diferentes universidades que integran el SUE, en su plan de desarrollo institucional la Universidad de Antioquia (2006) [9], centra su programa de responsabilidad social en el cubrimiento y apertura de nuevos cupos y expansión del servicio de educación en el departamento de Antioquia, extendiendo sus servicios por medio de la descentralización a nuevos municipios, en los cuales no existe acceso a la educación superior, y su inversión 
económica está destinada a la creación de estos nuevos centros de atención para estudiantes, dando nuevas oportunidades de crecimiento a los municipios que no cuentan con el servicio de formación en educación superior.

Tabla 1. Matriz de criterios de comparación

\begin{tabular}{|c|c|c|c|c|}
\hline CATEGORÍAS & $\begin{array}{l}\text { Campus } \\
\text { responsable }\end{array}$ & $\begin{array}{c}\text { Formación profesional } \\
\text { y ciudadana }\end{array}$ & Participación social & $\begin{array}{l}\text { Gestión social de } \\
\text { conocimiento }\end{array}$ \\
\hline Económica & $\begin{array}{c}\text { El buen gobierno y } \\
\text { el uso de recursos } \\
\text { enfocados al mejo- } \\
\text { ramiento del clima } \\
\text { organizacional con } \\
\text { responsabilidad am- } \\
\text { biental }\end{array}$ & $\begin{array}{l}\text { Esfuerzos económicos } \\
\text { enfocados al diseño } \\
\text { del mejoramiento de } \\
\text { condiciones sociales y } \\
\text { ambientales }\end{array}$ & $\begin{array}{l}\text { Direccionamiento de } \\
\text { esfuerzos financieros en } \\
\text { la participación de pro- } \\
\text { yectos con la comunidad, } \\
\text { para apuntar al desarrollo } \\
\text { social y ambiental }\end{array}$ & $\begin{array}{l}\text { Convocatorias de inves- } \\
\text { tigación que propicien el } \\
\text { desarrollo social e inviten } \\
\text { a la comunidad a ser par- } \\
\text { te activa de los mismos }\end{array}$ \\
\hline Legal & $\begin{array}{c}\text { Cumplimiento y } \\
\text { mejoramiento de } \\
\text { condiciones laborales } \\
\text { y académicas que } \\
\text { trasciendan los re- } \\
\text { querimientos legales } \\
\text { mínimos }\end{array}$ & $\begin{array}{l}\text { Normatividad y diseño } \\
\text { curricular que corres- } \\
\text { ponda a realidades } \\
\text { sociales }\end{array}$ & $\begin{array}{l}\text { Unión con sector público } \\
\text { y privado para la cons- } \\
\text { trucción de propuestas de } \\
\text { desarrollo social }\end{array}$ & $\begin{array}{l}\text { Factor investigativo para } \\
\text { el mejoramiento de la } \\
\text { calidad educativa }\end{array}$ \\
\hline Ética & $\begin{array}{l}\text { Promoción de com- } \\
\text { portamiento organi- } \\
\text { zacional socialmente } \\
\text { responsable }\end{array}$ & $\begin{array}{c}\text { Formación de profe- } \\
\text { sionales y ciudadanos } \\
\text { socialmente respon- } \\
\text { sables }\end{array}$ & $\begin{array}{l}\text { Conformación de comu- } \\
\text { nidades para promover el } \\
\text { intercambio constante de } \\
\text { conocimiento }\end{array}$ & $\begin{array}{l}\text { Mejoramiento curricular } \\
\text { conjunto entre la universi- } \\
\text { dad y la comunidad }\end{array}$ \\
\hline Discrecional & $\begin{array}{l}\text { Diseño democrático } \\
\text { de políticas que apo- } \\
\text { yen al mejoramiento } \\
\text { social y ambiental }\end{array}$ & $\begin{array}{c}\text { Profesionales compe- } \\
\text { tentes que promuevan } \\
\text { la solución de proble- } \\
\text { máticas sociales }\end{array}$ & $\begin{array}{l}\text { Participación en proyec- } \\
\text { tos externos a la insti- } \\
\text { tución, para aportar al } \\
\text { mejoramiento social }\end{array}$ & $\begin{array}{c}\text { Aprendizaje solidario } \\
\text { basado en proyectos } \\
\text { sociales }\end{array}$ \\
\hline
\end{tabular}

Debido a que los recursos propios de las IES, en su gran mayoría no son suficientes para suplir las necesidades propias de cada institución, las mismas recurren a alianzas estratégicas para poder cumplir con sus objetivos y planeaciones, integrando a actores del sector productivo, y la comunidad en general, tal es el caso de la Universidad Pedagógica y Tecnológica de Colombia (UPTC) [10] que en su planeación estratégica plantea el uso de alianzas para la formulación de políticas públicas, mediante las cuales se fortalezca el desarrollo comunitario local y regional en su zona de impacto, integrando al sector público y privado, con iniciativas de emprendimiento y productividad que representen empleo y bienestar para la región.

Por su parte la UMNG, al igual que las demás IES del SUE debe hacer anualmente una rendición publica de cuentas, contar con su Modelo Estándar de Control Interno y no tiene como objetivo la generación de utilidades. Adicionalmente, se recomienda considerar dentro de su presupuesto rubros para fortalecer las acciones requeridas para dar continuidad a los propósitos institucionales de contar con un campus responsable, fortalecer la formación 
ciudadana, y particularmente, fortalecer la participación social y gestión social del conocimiento que son los elementos más débiles actualmente.

\subsection{Legal}

Al ser reconocidas como instituciones de Educación superior por el Ministerio de Educación (MEN), las universidades estatales están reguladas y su planeación estratégica y planes de acción están enmarcados en la ley 30 de diciembre 28 de 1992 [7], en la que se organiza el sistema de educación superior en Colombia.

Así mismo, se otorga a las universidades la autonomía institucional, según el artículo 28 [7, pp. 5], en donde les reconoce el derecho a modificar sus estatutos, la designación de funciones administrativas y académicas, definir la planeación y diseño curricular, planificar los procesos formativos de los docentes, y diseñar los cronogramas investigativos, culturales y científicos; los procesos de admisión de los estudiantes, y administrar los recursos con el ánimo de cumplir con su objeto social.

En la mayoría de las universidades del SUE, se encuentra que la RSU no se entiende como un proceso integral transversal [4] y se confunde con extensión, por lo que se delega al departamento de proyección social y bienestar, el cual se encarga de manejar un ambiente adecuado para los integrantes de la comunidad educativa, sus iniciativas en la mayoria de los casos, y que se puede encontrar en la oferta de dicho departamento, se limita a actividades de bienestar para los estudiantes, que aportan al mejoramiento de la condición social, pero no cumplen con el fin ultimo de la RSU, que es brindar bienestar en varios aspectos a los diferentes actores de la comunidad educativa, y no solamente en el campo deportivo y recreacional.

Las universidades en sus mapas de procesos de Gestión de Calidad, resaltan la importancia de las funciones de la proyección social, tal es el caso de la Universidad de la Guajira, en donde se entiende la extensión como un proceso misional, certificado en Calidad bajo la norma ISO 9001, por medio del que se plantean diferentes líneas de trabajo, a partir de las necesidades de la región y formuladas bajo los objetivos de desarrollo de su zona de influencia. [11].

Los procesos de mejoramiento continuo son recurrentes en la planeación de las universidades, la realimentación de los diferentes procesos se ejecuta gracias a los sistemas de gestión de calidad en donde se recopila información, para que las directivas tomen decisiones de manera más acertada, como lo contempla la Universidad del Atlántico en su página web [12], donde aclaran su sistema integrado de gestión, está diseñado como un conjunto de estrategias que tienen como objetivo principal reconocer el impacto de la universidad en la sociedad y el medio ambiente, para definir procesos de mejoramiento continuo con impactos de bienestar para la comunidad en general. Por su parte, la UMNG cuenta con certificaciones en los sistemas de gestión de calidad: ISO 9001 y NTCGP 1000:2009, en el sistema de gestión de seguridad OHSAS 18001 y en el sistema de gestión ambiental ISO 14001. Y en su política integral "se compromete a cumplir con los requisitos aplicables asociados a sus grupos de interés pertinente con responsabilidad social" [13], cumpliendo de este modo con los requerimientos mandatorios que implican estos sistemas de gestión.

Es interesante encontrar que, solo una de las universidades estatales tiene en cuenta la norma ISO 26000:2010, mediante la cual se reconoce a las organizaciones por sus buenas prácticas en cuanto a Responsabilidad Social; la Universidad del Atlántico resalta [12]., desde el aspecto legal, que para la institución el cumplimiento de la norma ISO 26000:2010, es la definición de sí misma como una organización responsable de los impactos que genera en su actividad propia, y que estos están ligados a la sociedad y al medio ambiente, por lo cual es necesario plantear estándares de comportamiento ético y transparente, y en esta vía, aportar al desarrollo sostenible de la comunidad en general.

Al analizar los procesos adelantados por la Universidad de Caldas [14, pp. 79], en el sondeo entre la comunidad 
educativa, concluye la ingobernabilidad, anomia, y perdida de sentido son el eje fundamental de la problemática que diagnosticaron los docentes de planta de la institución; para ellos no hay normas, las instancias institucionales están determinadas desde los entes administrativos y gerenciales, a pesar que hay lineamientos dispuestos desde las directivas; el problema radica en que se encuentran escritos, pero en el diario vivir de la universidad ninguno se aplica.

Esto evidencia un caso de poca efectividad de las normas formuladas y la falta de interés por parte de los integrantes de la comunidad educativa, a pesar que formulen políticas y normas para cumplir con componentes de RSU.

En el país, el aseguramiento de la calidad esta regido por entidades que pertenecen al Ministerio de Educación, pero que tienen la función específica de impulsar y evaluar la calidad de la educación superior, de acuerdo con Cortés [15], desde cuya perspectiva se entiende el sistema de Aseguramiento de la calidad en la Educación Superior, como el proceso mediante el cual se acredita la calidad de nivel educativo, y que evalua a las diferentes instituciones por medio del apoyo del CONACES y el Consejo Nacional de acreditación (CNA).

Estos componentes han permitido que en los planes de desarrollo, sea donde principalmente se incluyan elementos que esten ligados a la RSU, aunque esto aparece como elemento de cumplimiento de las exigencias de acreditación para las universidades, componente tambien regulado por la ley 30 [6] y en el cual se nombra la RSU como un requisito planteado en el documento de lineamientos para la acreditación institucional en el númeral 10-7 [16], en el que se concluye que una institución de alta calidad es reconocida como tal, cuando se identifica que las politicas y los programas diseñados para la oferta académica, están enfocados en el compromiso con el entorno propio, interactuando constantemente con el contexto y los lugares donde tiene presencia la universidad.

La investigación cumple un papel protagónico en este aspecto, puesto que tal como lo reconoce la universidad Pedagógica en su observatorio de Responsabilidad social [17], en donde se encuentra la necesidad de enfocar los esfuerzos de carácter investigativo en el diseño y desarrollo de programas que esten orientados al bienestar de la comunidad, identificando problematicas en el contexto, con estudios en grupos sociales que relacionados con la comunidad educativa; las lineas de investigación están ligadas al plan de desarrollo de la universidad, y los proyectos y programas son diseñados y puestos en marcha directamente por los estudiantes, quienes ven fortalecido su proceso de formación profesional gracias a la experiencia investigativa en estudios que incorporan directamente a la sociedad.

En el cumplimiento de sus funciones misionales la UMNG, de acuerdo a lo encontrado por M.P Padilla [18], ha adelantado distintas acciones en las tres funciones sustativas de la educación superior:

1. Docencia: La formación permanente del personal docente se presenta por medio de capacitación y programas diseñados para el crecimiento personal y profesional del capital humano, realzando las capacidades de los docentes frente al cumplimiento misional de la universidad, y teniendo en cuenta el diseño de los perfiles de docentes, que generen espacios de innovación constante frente a las necesidades del mercado.

2. Investigación: Los procesos investigativos están diseñados para potenciar la creación e innovación tecnologica en diferentes campos, de tal forma que, las convocatorias se enfocan en el apoyo a este tipo de iniciativas, propiciando el desarrollo cientifico y nuevas prácticas en la institución.

3. Extensión y proyección social: La comunidad académica está comprometida con la ejecución de prácticas que identifican problematicas de la sociedad, de esta manera, se generan espacios de intercambio de experiencias a nivel nacional e internacional, y en las que a diario se reflexione de manera colectiva y se fortalezca los 
componentes éticos y humanos de la organización.

Se entiende entonces que la UMNG al contar con acreditación institucional cumple con éste y los demás requerimientos establecidos por el Consejo Nacional de Acreditación, satisfaciendo de esta manera su compromiso de ofrecer educación de calidad. Sin embargo, se deben contar con políticas especificas que conduzcan a normatizar para fortalecer la participación social y la gestión social del conocimiento.

\section{3. Ética}

La facultad de Ingeniería de la Universidad Nacional [19] hace referencia a la RSU, como un tema que ha tomado cierta importancia dentro de la universidad, pero que no existe un acuerdo sobre el mismo, puesto que falta un reglamento o una normativa que permita implementar acciones de manera sistemática y transversal en los diferentes procesos que se llevan a diario dentro de la universidad; el componente de obligatoriedad que contiene la misma RSU, se convierte en un limitante para la ejecución plena de la misma, dado que los integrantes de la facultad identifican a la RSU, como algo más que se debe cumplir, y no como un compromiso que se adquiere a nivel personal y alineado con la institución. Es necesario limitar el rigor académico para la definición de la RSU, para brindar lineamientos claros a la comunidad, como resultado, se potencializa el cumplimiento de la RSU en la facultad, y su extensión a nivel institucional.

Se puede evidenciar que la universidad no tiene claro como incluir la responsabilidad social dentro de sus acciones y planificación, y a pesar de tener la intención de incluirla en todos sus procesos, no existen directrices que guíen el cumplimiento de las políticas que pueden surgir en cuanto a RSU.

Esta situación se extiende al resto de universidades del SUE, y se evidencia en la inclusión de la RSU simplemente como un valor institucional el cual tiene el papel de cumplimiento de una norma para la acreditación de la universidad, y que por ser valores institucionales se supone están contemplados dentro de la misión y visión de la organización, tal como se mencionó anteriormente en el aspecto legal.

Para el mejoramiento de los procesos en RSU, la Universidad Pedagógica en su componente ético, cuenta con un observatorio de responsabilidad social y una estrategia clara, como lo evidencia en el informe de dicho observatorio [17], en donde se define al estudiante como un individuo que ingresa a la universidad con unos presaberes que se modifican o moldean de acuerdo al proceso educativo y formativo desarrollado durante su estancia de la universidad; a partir del departamento de bienestar universitario se busca optimizar el conocimiento previo de los estudiantes, por medio de la practica docente, teniendo en cuenta que este caustro cumple el rol de educadora de educadores, por lo tanto se genera una base de datos mediante la cual se identifican problemáticas reales de la comunidad y se caracteriza la población estudiantil y lo que permite generar procesos de bienestar de manera sistemática y mejorar la toma de decisiones en la formulación de políticas de bienestar en el corto, mediano y largo plazo que cubran las expectativas de la comunidad estudiantil.

En este apartado, se identifica como, desde su objeto de formadora de educadores, la Universidad Pedagógica dedica sus esfuerzos a reconocer las diferencias de cada uno de los integrantes de la comunicadad educativa, en especial, de sus estudiantes. Esto permite a la universidad actuar y asegurar que el docente guie su labor a la formación de acuerdo a las necesidades de los estudiantes; y que los estudiantes, al entrar en contacto con temas de investigación ligados a su propia realidad, sean agentes de mejoramiento de condiciones sociales de su entorno gracias a los esfuerzos que se adelantan por parte de la misma.

Un factor determinante en este tema, es el surgimiento de posconflicto, en el cual la cátedra de la paz, significa un compromiso por parte de la educación superior, para formar profesionales que puedan crear espacios productivos 
en un país que no esta en conflicto y, por lo tanto, tiene mayores oportunidades de explotación de recursos. No obstante, estos ser manejados conscientemente, siendo racionales en el uso de los mismos y pensando en el futuro. Se hace relevante contar con un capital humano abierto al diálogo, al perdón, la tolerancia y la aceptación de las diferencias étnicas, culturales y de pensamiento, tal como lo promueve la Universidad del Cauca [20, pp. 2] en su plan de desarrollo en el que plantea que la equidad, la transparencia, y diferentes valores que propenden por el respeto y la calidad de la vida de los estudiantes; valores considerados desde la misión institucional, y desde la cual se busca crear espacios de integración, desarrollo comunitario, tolerancia, convivencia e integridad humana.

Este último elemento juega un papel importante considerando el rol que tiene el sector defensa en la UMNG [2]. Se propone que la actuación ética permee la ejecución de las actividades cotidianas y el cumplimiento de las funciones misionales establecidas por la Ley general de educación [6]: docencia, investigación y extensión, al igual que en la gestión de manera transversal.

\subsection{Discrecional}

En lo encontrado para cada una de las instituciones, cabe resaltar que, principalmente, se busca la formación de estudiantes con proyección internacional, por lo tanto, la formación esta acompañada desde el área académica y el componenete administrativo, conjugando actividades que promuevan en el estudiante habilidades que le permitan desempeñarse en cualquier contexto de manera versátil. Tal como ocurre en la Universidad de Nariño, en la que se plantea [21, pp. 52] que la proyección social debe diseñar proyectos que contribuyan al mejoramiento de condiciones sociales concretas, por medio de la interacción con sectores económicos y sociales tanto públicos como privados.

En los documentos de planeación, se encuentran componenetes en los cuales se hace énfasis en la relación de la academia con el sector productivo, el estado y la sociedad; por lo tanto, los esfuerzos de proyección investigativa están ligados a proyectos que involucren a estos actores, y que respondan a las necesidades que plantean cada uno de estos.

Para llevar a cabo este tipo de iniciativas, es importante que los docentes y el cuerpo administrativo, este capacitado para poder responder a estas exigencias. En este sentido, es necesario recurrir a distintas alternativas para cumplir con este propósito, como lo son las alianzas estratégicas, que resultan ser una gran oportunidad para alcanzarlos, ya que los recursos económicos propios no son suficientes para cumplir estos objetivos.

Para la extensión y la proyección social, una labor importante es el seguimiento que se hace a los egresados, el conocimiento del tiempo de vinculación laboral, la remuneración promedio y la continuidad en la formación académica. Esto con el fin de medir la calidad de la educación que reciben del alma mater. Está función es necesaria en todas las universidades, puesto que es un requisito para la acreditación, tanto institucional como de programas, pero más allá, es un buen indicador de calidad de la educación que reciben los estudiantes. En la UMNG se integran en la proyección social, las prácticas de los estudiantes con el sector productivo para realizar la realimentación de las experiencias de los graduandos.

El conocimiento de las condiciones propias del individuo, coadyuvan al mejoramiento de las condiciones éticas y humanas de los estudiantes, por lo tanto, en la UMNG, la interacción con agentes externos a la universidad permite extender prácticas que motiven y expandan el buen actuar de toda la comunidad en el nivel ético y moral, tal como se plantea en su proyección social [13].

La UMNG adelanta actividades para el beneficio de la sociedad, en las que se integran los diferentes actores de la comunidad, en pro del mejoramiento de las condiciones de formación de los estudiantes, en sus palabras:

"La universidad cuenta con programas de formación avanzada y capacitación para el trabajo y el desarrollo 
Humano lo cual permite avanzar en el proceso de crecimiento y cualificación profesional determinando a partir del conjunto de las competencias institucionales, el perfil necesario de docentes, con el fin de focalizar el quehacer y la transformación institucional en la generación de cambios y de prácticas innovadoras" [13].

Aunque este elemento no es mandatorio, se propone contar con una atención especial al beneficio del sector defensa que corresponde a un stakeholder exclusivo de la UMNG.

\section{CONCLUSIONES}

El análisis comparativo matricial propuesto como punto de partida para la construcción de modelos propios de RSU favorece la integración de los conceptos tradicionales de Responsabilidad Social Empresarial y Responsabilidad Social Universitaria, al entender las categorías como complementarias, así las cosas se tendrían dieciséis (16) elementos de valoración que resultan del cruce de las categorías: Campus responsable, Formación profesional y ciudadana, Participación social y Gestión social del conocimiento con las categorías: Económica, Legal, Ética y Discrecional.

La RSU para las universidades públicas se presenta como un elemento integrado a los programas de extensión y proyección social, los cuales son tenidos en cuenta en los procesos de acreditación por parte del Ministerio de Educación Nacional. Sin embargo, existen dentro de las mismas iniciativas en pro del cumplimiento del objeto social de la universidad de formar ciudadanos con conciencia social, por lo tanto, los procesos académicos deben apuntar a fortalecer el componente de responsabilidad social de sus educandos.

Las limitaciones económicas de las IES públicas, entorpecen el cumplimiento de los procesos y planteamientos hechos por las directivas en lo que refiere a RSU. Como consecuencia, estas deben recurrir a estrategias que permitan reforzar dichas actividades, entre las cuales se encuentran alianzas estratégicas con entidades del sector privado.

La capacitación de docentes, descentralización de actividades y ampliación de la cobertura de la oferta educativa, son iniciativas reconocidas por las IES en el mejoramiento de condiciones y cumplimiento de la RSU, al igual que la integración de la proyección social, extensión y el bienestar universitario dentro de los programas y planes de mejoramiento.

Se reconoce como un elemento de calidad, la inclusión e implementación de la RSU dentro de los planes de desarrollo de las instituciones universitarias.

El proceso de posconflicto significa un reto para las IES, y debe estar contemplado dentro del proceso de planeación en la RSU, ya que es preciso reconocer las necesidades de formación de los involucrados en dicho proceso, para que la educación que se oferte responda a la realidad de la comunidad a la cual pertenece la institución.

A pesar de los esfuerzos de las instituciones, es necesario que sus directivos cuenten con el conocimiento suficiente para poder diseñar e implementar las iniciativas que se relacionan con la RSU, para que estas al momento de ser ejecutadas sean mucho más efectivas dentro de la comunidad.

Lograr contar con un campus responsable, con formación profesional y ciudadana, tener participación social y gestión social del conocimiento requiere como mínimo un compromiso institucional que forme parte de las políticas y reglamentos, de la aplicación de la ética de manera transversal en el desarrollo de las funciones misionales (docencia, investigación, extensión y gestión) y que se vea reflejado en la planeación económica mediante la elaboración y aprobación del presupuesto con rubros específicos para tales fines. Se propone en el modelo de RSU dar atención especial al sector defensa que corresponde a un stakeholder exclusivo de la UMNG.

Como investigaciones futuras, en esta misma línea, se 
propone consultar las expectativas de las partes interesadas (internas y externas) con respecto a un modelo de RSU, de modo que, su formulación no sólo integre las acciones de las IES públicas al respecto, sino que, además, responda a las expectativas de todos los miembros e involucrados con la comunidad neogranadina.

\section{AGRADECIMIENTOS}

Producto derivado del proyecto INV-ING-2481 financiado por la Vicerrectoría de Investigaciones de la Universidad Militar Nueva Granada- Vigencia 2017.

\section{REFERENCIAS}

[1] J. R García. (2003). "La formación de ciudadanos: la escuela, un escenario posible". Revista Latinoamericana de Ciencias Sociales, Niñez y Juventud. [En Linea]. 1(2), pp. 115 -143. Diponible: http://www. scielo.org.co/scielo.php?script=sci_arttext\&pi$d=$ S1692-715X2003000200005

[2] Universidad Militar Nueva Granada. "Misión, Visión y Objetivos". [En linea]. Disponible: http://www. umng.edu.co/la-universidad/mvo

[3] Universidad Militar Nueva Granda, Plan de desarroIlo institucional 2009-2029. Bogotá, 2009.

[4] Vallaeys, F., De La Cruz, C., Sasia, P., Camdessus, I. (2009). Responsabilidad social universitaria: manual de primeros pasos. Washington: Banco Interamericano de Desarrollo. Editorial McGraw-Hill.

[5] J. P. Sulbarán, "El concepto de Responsabilidad Social dela Empresa", Revista Economía, No. 10, pp. 225-249, 1995.

[6] Abla A.H. Bokhari, “Universities' Social Responsibility (USR) and Sustainable Development: A Conceptual Framework", SSRG International Journal of Economics and Management Studies, pp. 1-12. 2017.

[7] Congreso de la Republica de Colombia, Ley 30: Por la cual se organiza el servicio público de la educación superior. Bogotá, Diario Oficial, 1992.

[8] Sistema Universitario Estatal, Declaración Sistema Universitario Estatal. Pasto, 2016.
[9] Universidad de Antioquia, Plan de desarrollo 20062016. Medellin, 2006.

[10] Universidad Pedagógica y Tecnológica de Colombia, Plan de Desarrolllo Institucional 2014-2018. Tunja, 2014.

[11] Universidad de la Guajira, "Centro de extensión". [En linea]. Disponible: http://www.uniguajira.edu. co/sobre-nosotros-centro-extension.

[12] Universidad del Atlántico, "Responsabilidad social universitaria RSU". [En linea]. Disponible: https:// www.uniatlantico.edu.co/uatlantico/administrativa/administrativa-RSU.

[13] Universidad Militar Nueva Granada. Política integral". [En linea]. Disponible: http://www.umng. edu.co/web/guest/la-universidad//division-de-gestion-de-calidad/sistema-de-gestion-de-calidad

[14] Universidad de Caldas, Plan de desarrollo institucional. Manizales, 2008.

[15] J. L. Cortés, Estudio comparativo de los sistemas de gestión en las Universidades Públicas de Bogotá. Bogotá, 2017.

[16] Consejo Nacional de Acreditación, Lineamientos para la acreditación Institucional. Bogotá, 2015.

[17] Universidad Pedagógica Nacional, Responsabilidad Social Universitaria Como sistema de Investigación, caracterización, evaluación y sistematización de información. Bogotá, 2012.

[18] M. P. Padilla, "Acciones realizadas por la UMNG para la incorporación de la responsabilidad social universitaria", Trabajo de fin de especialización, Facultad de estudios a distancia, Universidad Militar Nueva Granada, Bogotá, 2017.

[19] H. G. Cortés, "Responsabilidad Social Universitaria. Una mirada a la Universidad Nacional de Colombia", Tesis de Maestria, Universidad Nacional de Colombia, Bogotá, 2012.

[20] Universidad del Cauca, Sistema de Cultura y Bienestar. Popayan, 2015.

[21] Universidad de Nariño, Plan de Desarrollo UNAR 2008-2020. Bogotá, 2007 